INTERFACE SCIENCE 1, 223-235 (1993).

(C) Kluwer Academic Publishers, Boston. Manufactured in The Netherlands.

\title{
Adhesion at a Heterophase Interface: First-Principles Study of $\mathrm{Mo}(001) / \mathrm{MoSi}_{2}(001)$
}

\author{
T. HONG \\ Department of Materials Science and Engineering, University of Michigan, Ann Arbor, MI 48109-2136
}

J.R. SMITH

Physics Department, General Motors Research and Development Center, Warren, MI 48090-9055

D.J. SROLOVITZ

Department of Materials Science and Engineering, University of Michigan, Ann Arbor, MI 48109-2136

Received May 3, 1993; Revised August 4, 1993

Keywords: Adhesion, heterophase interface, first-principles electronic structure calculations.

\begin{abstract}
The bonding characteristics, interfacial energetics, and electronic structure associated with adhesion at the $\mathrm{Mo}^{-\mathrm{MoSi}_{2}(001)}$ heterophase interface are investigated using the first-principles, selfconsistent local orbital method. We found both the adhesive energy and peak interfacial strength for the interface to be $10 \%-15 \%$ smaller than the respective values for cleavage along the (001) planes in crystalline Mo and $\mathrm{MoSi}_{2}$. The equilibrium interlayer separation between $\mathrm{Mo}$ and $\mathrm{MoSi}_{2}$ is found to lie between the interplanar spacings of crystalline Mo and $\mathrm{MoSi}_{2}$. The interfacial adhesive bonding is attributable to the combination of a nearly uniform band of charge accumulation at the interface and directional charge accumulation between atoms across the interface. These first-principles calculations demonstrate that the universal-binding-energy relation can be extended to describe adhesion between dissimilar materials.
\end{abstract}

\section{Introduction}

The adhesive energy or work of adhesion of an interface is the energy required to separate the two materials that meet at the interface, in the absence of any dissipative processes (such as dislocation motion). In this sense, the adhesive energy is the fundamental quantity which describes the strength of the bond between two materials. It is this adhesive energy that determines the fracture properties of materials within the simple thermodynamic model of brittle fracture due to Griffith [1]. While more modern theories of interfacial fracture are capable of including a range of other physical phenomena (e.g., plasticity, segregation, etc.), they all require, at their most fundamental level, a description of the en- ergy or force required to separate the material at the interface-i.e., the adhesive energy. As a result of the centrality of the adhesive properties in fracture, the adhesive energy becomes a focal point of an increasing number of studies involving physicists, chemists, and materials scientists. As the search for high-performance materials continues, it is common to combine two or more materials with complimentary properties in order to optimize properties. This has led to considerable interest in the fracture behavior of composites and the interfaces between the dissimilar materials within them. The present paper focuses on the adhesive energetics of heterophase interfaces in a metal/intermetallic composite. This work is part of an ongoing effort to obtain a microscopic understanding of the mech- 
anisms that control adhesion in order to provide a rational basis for the design of increasingly complex materials.

Several approaches can be applied to theoretically determine the adhesive energy of heterophase interfaces. These include empirical methods, semiempirical methods and $a b$ initio methods. In the first category are atomistic simulations based upon physically motivated, empirical interatomic potentials. The advantage of this approach is that atomistic simulations of this type are computationally efficient and can be used to obtain optimized (equilibrium) atomic positions. On the other hand, this efficiency comes at the cost of accuracy as the interatomic potentials do not typically yield accurate bonding energetics in very different environments from that in which they were fitted. The $a b$ initio or first-principles methods are fully quantum mechanical and yield reliable bonding energetics and electronic structure. These methods are generally not sufficiently computationally efficient to allow optimization of atomic positions. Semiempirical methods combine the advantages and disadvantages of each. In the present study, we focus on a heterophase interface where relatively little atomic relaxation is expected. In this case, the accuracy of the $a b$ initio methods are not severely compromised by the lack of atomic relaxation.

Heterophase interfaces, by their nature, involve systems with periodicity broken in the direction perpendicular to the interface. The lowered symmetry normally requires large unit cells in order to reasonably represent the interface. While modern, state-of-the-art first-principles calculations (within the framework of the density functional theory [2]) have been shown to accurately predict a wide variety of phenomena in a wide range of materials, the substantial supercomputing resources they require have prevented their widespread application in investigations of adhesive properties of heterophase interfaces. To date, there has not been a first-principles study which has provided adhesive energies for heterophase interfaces between metallic materials.

In this paper, we report the results of a fully three-dimensional, first-principles study of adhesion at a heterophase interface. The electronic structure and total energy of the Mo(001)$\mathrm{MoSi}_{2}(001)$ interface is calculated as a function of interfacial spacing by using the first-principles, self-consistent local orbital (SCLO) method [3]. To determine the adhesive energetics, the recently proposed four-point method [4] is then employed to fit the calculated energy values at different interfacial separations to a universalbinding-energy relation (UBER) [5]. The ideal work of adhesion (or adhesive energy), the peak interfacial strength (the peak derivative of the energy with respect to the separation of the solids at the interface), and the full adhesion curve are all obtained by performing calculations at as few as four interfacial separations. The adhesive energy and the pertinent surface energies are combined to analyze the relative configurational stability of different possible interfacial geometries.

$\mathrm{MoSi}_{2}$ is of particular interest for hightemperature structural applications [6]. The inherently low ductility of pure $\mathrm{MoSi}_{2}$ has led several groups [6] to create a composite of $\mathrm{MoSi}_{2}$ and other tougher metals (such as $\mathrm{Mo}$ and $\mathrm{Nb}$ ) in order to achieve higher ductility through ductile phase toughening. $\mathrm{MoSi}_{2}$ crystallizes in the bodycentered tetragonal structure with experimental lattice constants [7] $a=3.202 \AA, c=7.851 \AA$. The experimental lattice constant of bcc Mo is [8] $a=3.14 \AA$ and, hence, the lattice mismatch for the $[100] / /[100](001)$ planes of the two materials is then less than $2 \%$. Hence, we expect the interface to consist of epitaxially bonded Mo and $\mathrm{MoSi}_{2}$ with a periodic array of dislocations at the interface which are spaced approximately $160 \AA$ apart. In between these widely separated dislocations, we expect little atomic relaxation due to the epitaxial constraints and the short screening lengths in metals. In the present study, we consider the $[100] / /[100]$ (001) epitaxial interface between $\mathrm{Mo}$ and $\mathrm{MoSi}_{2}$ which may be viewed as either the state of the interface for very thin films (smaller than the critical thickness for dislocation formation) or as the region between the misfit dislocations when the two materials are thick. In this sense, this is an ideal candidate metal/intermetallic system for studying heterophase interfaces using first-principles methods.

\section{Method}

The electronic structures and total energies of all of the interfacial configurations considered 


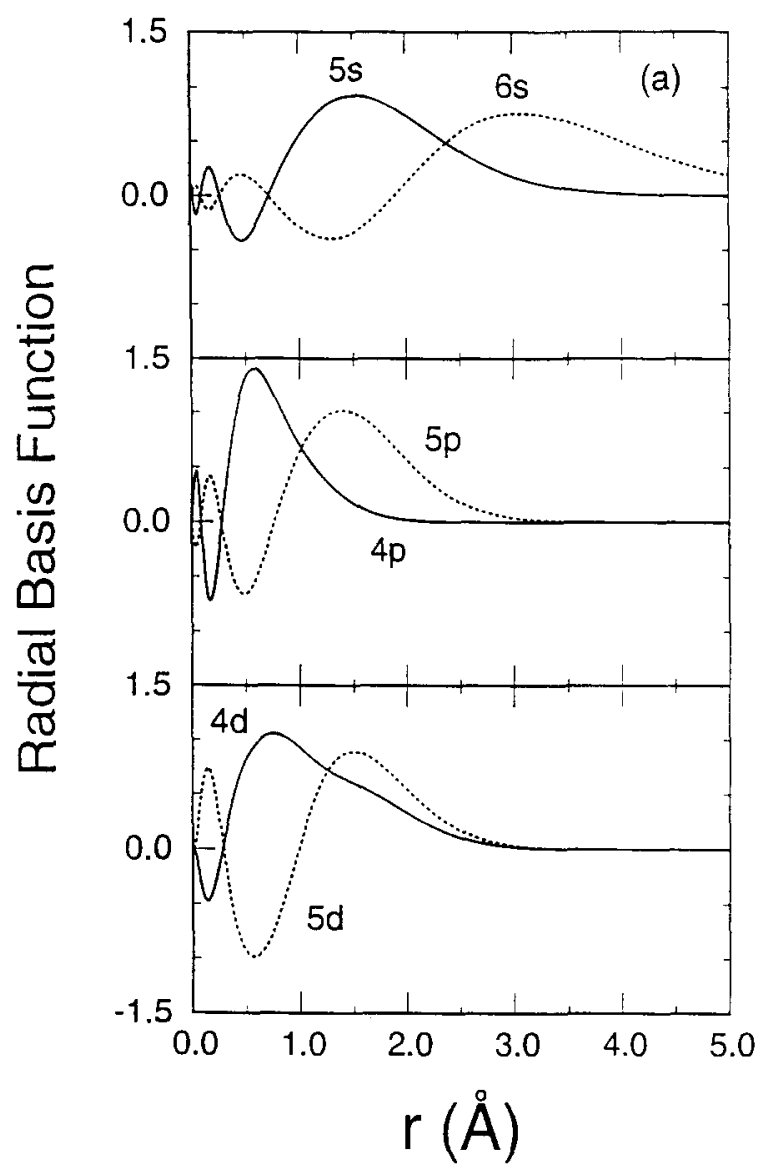

(a)

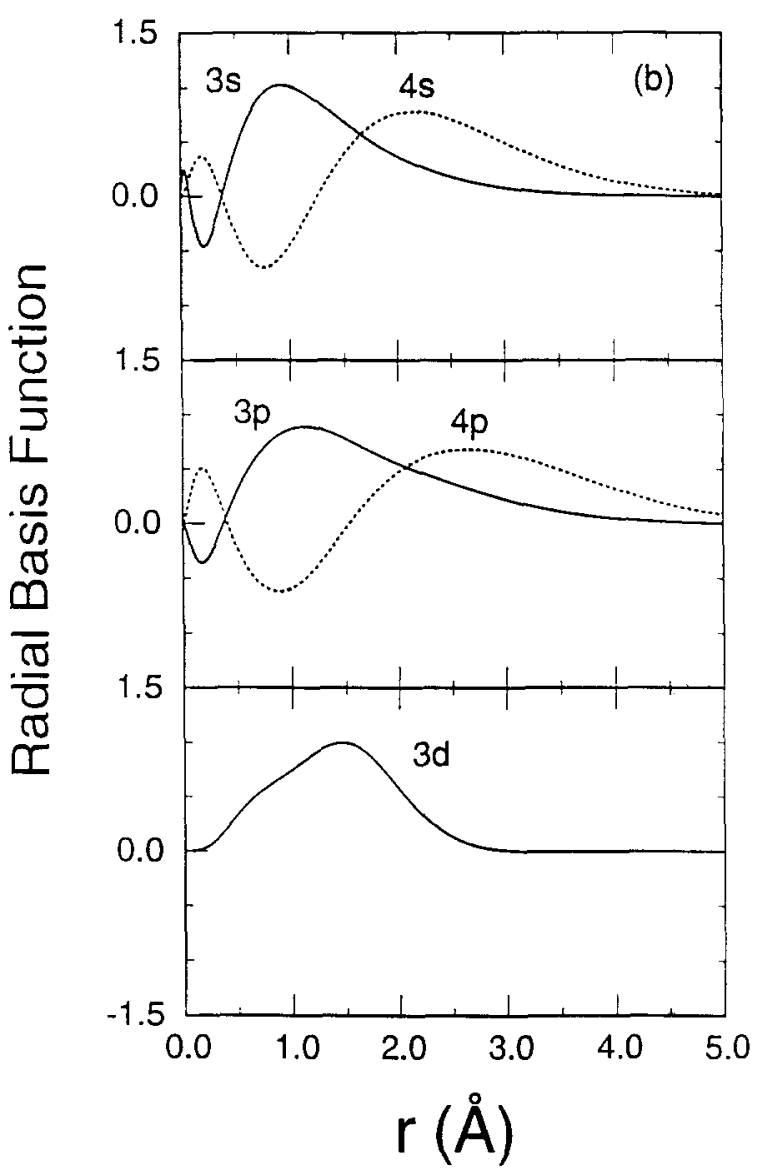

(b)

Figure 1. Radial basis functions ( $r$ times the radial wave function) plotted versus $r$ for the outer orbitals of (a) Mo; and (b) Si.

in this study are determined using the SCLO method [3] which has previously been successfully applied to the study of transition metal surfaces. The Ceperley-Alder [9] form of the exchangecorrelation potential is used in the local-densityfunctional single-particle equation [2]. The selfconsistent iterations are carried out until electron eigenvalues are converged to better than $5 \mathrm{MeV}$. A vacuum space with a thickness of four times the lattice constant of bcc Mo is added to both sides of the slabs to form three-dimensional unit cells. This was found to greatly improve computational efficiency [3]. The localized basis set includes all of the core and valence orbitals. The core orbitals are represented by linear combinations of Gaussians, which approximate the atomic wave functions found by solving the Kohn-Sham equations for the atoms. For the valence orbitals, a minimum basis which, for efficiency, might approximate contracted atomic wave functions is augmented by more diffuse orbitals. The basis set constructed in this manner contains much of the flexibility of the quantum chemist's doublezeta-plus-polarization basis sets. Plots of the radial parts of the conduction-band local orbitals of Mo and Si are exhibited in figure 1(a) and (b), respectively.

The prescription used to obtain the basis set of Mo is the same as that used for other transition metals [3]. The $4 p$ and $4 d$ valence orbitals of Mo are fitted in the same way as the core orbitals. The $5 s$ valence orbital is, however, fitted to a contracted atomic $5 s$ wave function for the sake of computational efficiency. In addition to the minimum basis set of $4 p, 4 d$, and $5 s$ orbitals, more diffuse $5 p, 5 d$, and $6 s$ orbitals are also 
used. The $5 d$ orbital is made up of the most diffuse Gaussian of the inner $d$-symmetry orbitals plus the appropriate linear combination which orthogonalizes the $5 d$ orbital to the $3 d$ and $4 d$ orbitals. Similarly, the $5 p$ and $6 s$ orbitals are each constructed from a single diffuse Gaussian with additional terms orthogonalizing them to the inner orbitals of their respective symmetry. The inclusion of the more diffuse $6 s$ orbital makes it possible to contract the inner $5 s$ orbital, which greatly reduces the number of matrix elements that need to be included [3]. The scale factor for the diffuse $5 p$ orbital Gaussian was chosen to be 0.2 ; a value used in a number of previous calculations of transition metals [10]. For the $6 s$ orbital, the scale factor was determined by minimizing the total energy of a three-layer slab of bcc Mo. The optimized value is found to be 0.04. This is exactly what was found for bcc Fe in previous studies [10].

The procedure for obtaining the basis set of Si is somewhat different. Si differs from the transition metals in that it does not contain $d$ electrons. Orbitals up to $3 s$ and $3 p$ are fitted to approximate the unmodified atomic wave functions. The $4 s$ and $4 p$ orbitals, on the other hand, each consist of a single diffuse Gaussian orthogonalized to the inner orbitals. The $4 s$ and $4 p$ orbital scale factors are simultaneously determined by minimizing the total energy of a three-layer slab of $\mathrm{MoSi}_{2}$. The $3 d$ orbital is fitted to the contracted atomic $3 d$ wave function computed self-consistently for a $\mathrm{Si}$ atom in the atomic configuration $3 s^{1} 3 p^{2} 3 d^{1}$. This is done to ensure that the $3 d$ orbital has relatively short range in order to lessen the possibility of linear dependencies-a common problem in the calculations involving nonorthogonal basis sets.

As seen in figures 1(a) and (b), all of the fitted orbitals, except the Mo $4 d$ and $\mathrm{Si} 3 d$, are quite smooth. Further investigation has shown that the slight undulations of the Mo $4 d$ and $\mathrm{Si} 3 d$ are due to the limited number of Gaussians used to fit these orbitals. Test calculations found that the electronic structure and total energy results were nearly unchanged when orbitals fitted with more Gaussians (to eliminate the undulations of the Mo $4 d$ and $\mathrm{Si} 3 d$ orbitals) were used. Since marginal gains in accuracy are achieved and greater computational effort is required when orbitals fitted with more Gaussians are employed, we chose to use the Mo $4 d$ and Si $3 d$ orbitals as shown in figures $1(\mathrm{a})$ and (b).

The total energy as a function of $\mathrm{Mo}-\mathrm{MoSi}_{2}$ interfacial separation was fitted to the universal binding energy relation (UBER) using the four-point method [4]. The adhesive energy per unit surface area $(E)$ as a function of interfacial separation $(d)$ is thus fitted to

$$
E=-E_{0}\left(1+a^{*}\right) e^{-a^{*}}
$$

where

$$
a^{*}=\left(d-d_{0}\right) / l
$$

The equilibrium interfacial separation is denoted by $d_{0}$ and the corresponding adhesive energy is $-E_{0}$ ( $E_{0}$ will be referred to as the ideal adhesive energy hereafter). The zero of the adhesive energy is chosen to correspond with effectively infinite separation. The scaling length $l$ in equation (2) is a fitting parameter which provides a measure of the elastic characteristics of the material [5]. The interfacial strength $\sigma$ is defined as the derivative of the total energy with respect to the interfacial separation $d$ and is, found from equations (1) and (2),

$$
\sigma=\sigma_{\max } a^{*} e^{\left(1-a^{*}\right)}
$$

where the ideal peak interfacial strength $\sigma_{\max }$ is related to the ideal adhesive energy $E_{0}$ by

$$
\sigma_{\max }=\frac{2 E_{0}}{l e}
$$

and where $e$ is the base of the natural logarithm. The factor of two accounts for the fact that traditionally $\sigma$ is considered to be a force per cross-sectional area, while $E_{0}$ is an energy per surface area.

In order to simplify the calculations, the Mo$\mathrm{MoSi}_{2}$ interface is assumed to be epitaxial with a lattice constant of $a=3.202 \AA$, the $\mathrm{MoSi}_{2}$ lattice constant. The interlayer separation in the Mo slab was chosen to be half of the experimental lattice constant of bcc Mo [8]. The separation between the $\mathrm{Si}-\mathrm{Si}$ and $\mathrm{Mo}-\mathrm{Si}$ planes in $\mathrm{MoSi}_{2}$ were set equal to each other $(=c / 6)$. This has been shown to be a good approximation [7, 11]. Only very small atomic relaxation are expected and hence, for simplicity, no atomic relaxation and reconstructions are considered. 
Table 1. Calculated ideal adhesive energy $\left(E_{0}\right)$ peak interfacial strength $\left(\sigma_{\max }\right)$, equilibrium separation $\left(d_{0}\right)$, and the scaling length $(l)$ for four different $\mathrm{MoMoSi}_{2}$ configurations and for perfect $\mathrm{MoSi}_{2}$ crystals. Stacking configurations all start at the mirror plane, while actual slabs contain twice the number of layers shown.

\begin{tabular}{lcccccc}
\hline & MoMo/SiMoSi & MoMo/SiMoSiSi & MoMoMo/SiMoSiSi & MoMo/SiSiMoSi & MoSi/SiMoSi & MoSiSi/MoSiSi \\
\hline$E_{0}\left(\mathrm{~mJ} / \mathrm{m}^{2}\right)$ & 4,100 & 3,590 & 3,500 & 4,610 & 3,860 & 4,600 \\
\hline$\sigma_{\max }(\mathrm{GPa})$ & 51.6 & 40.8 & 39.6 & 53.0 & 43.8 & 49.4 \\
\hline$d_{0}(\AA)$ & 1.37 & 1.44 & 1.44 & 1.36 & 1.28 & 1.36 \\
\hline$l(\AA)$ & 0.59 & 0.65 & 0.65 & 0.64 & 0.65 & 0.68 \\
\hline
\end{tabular}

\section{Results}

Depending upon the stacking sequence near the interface, three distinct interfacial arrangements are possible. These arrangements can be represented by MoMo//MoSiSi, MoMo//SiMoSi, and MoMo//SiSiMo, where the double slashes are used to denote the interface. While only those atomic layers closest to the interface that are needed to distinguish the interfacial arrangements are listed, one might also wonder how thick the films should be to adequately represent adhesion. Both $\mathrm{Mo}$ and $\mathrm{MoSi}_{2}$ are metallic. Since short electronic screening lengths are characteristic of metallic systems, effectively limiting interfacial electronic effects to one or two layers of the interface, it is reasonable to model the interface by slabs containing a few atomic layers. That this is valid will be clear from electron density distributions that will be shown later in this paper.

In order to examine the dependence of interfacial properties upon the thickness of the slabs and interfacial arrangement, four different Mo$\mathrm{MoSi}_{2}$ interfacial configurations are considered in this study. To facilitate the separation of the Hamiltonian matrix into those with even and odd reflection symmetry and to keep all of the matrix elements real, reflection symmetry in the direction perpendicular to the interface is maintained in all configurations. The stacking sequences of the four configurations starting from the mirror plane are $\mathrm{MoMo} / / \mathrm{SiMoSi}$, MoMo//SiMoSiSi, MoMoMo//SiMoSiSi, and MoMo//SiSiMoSi, respectively. The first three configurations all correspond to arrangement where the first $\mathrm{MoSi}_{2}$ layer is Si and the second is Mo but with different Mo and $\mathrm{MoSi}_{2}$ slab thicknesses. The last config- uration corresponds to the arrangement in which the first two $\mathrm{MoSi}_{2}$ layers adjacent to the interface are $\mathrm{Si}$. For purposes of comparison, results for ideal adhesion in pure $\mathrm{MoSi}_{2}$, cleaved along a (001) plane are also reported. The two possible $\mathrm{MoSi}_{2}$ (001) cleavage planes are $\mathrm{MoSi} / / \mathrm{SiMoSi}$ and MoSiSi//MoSiSi. The MoMo/MoSiSi interfacial arrangement is not included in this study since this case will be nearly indistinguishable from that of cleavage in pure Mo.

\subsection{Adhesion}

The calculated values of the ideal adhesive energy $\left(E_{0}\right)$, the peak interfacial strength $\left(\sigma_{\max }\right)$, the equilibrium interfacial separation $\left(d_{0}\right)$, and the scaling length $(l)$ for all six interfacial geometrics described above are reported in table 1 . By comparing the adhesion results for the three geometrics in which a single Si layer is at the interface, it is clear that the thickness of slabs does not substantially affect the ideal adhesive energy, the peak interfacial strength, or the equilibrium interfacial separation. The MoMo//SiMoSiSi and MoMoMo//SiMoSiSi $E_{0}, \sigma_{\max }$ and $d_{0}$ all agree to within $3 \%$. This indicates that a three-layer slab is adequate for modeling bulk Mo. This is a direct manifestation of the very short screening length in metals. The somewhat larger differences between the results with two different $\mathrm{MoSi}_{2}$ slab thicknesses, on the other hand, are largely due to the smaller interplanar spacing in $\mathrm{MoSi}_{2}$ than in Mo (approximately 15\%) in the [001] direction. Based upon the above discussion, we assume that a slab of $\mathrm{MoSi}_{2}$ of four layers is sufficient to represent the bulk $\mathrm{MoSi}_{2}$.

It is not possible, based upon the data in ta- 


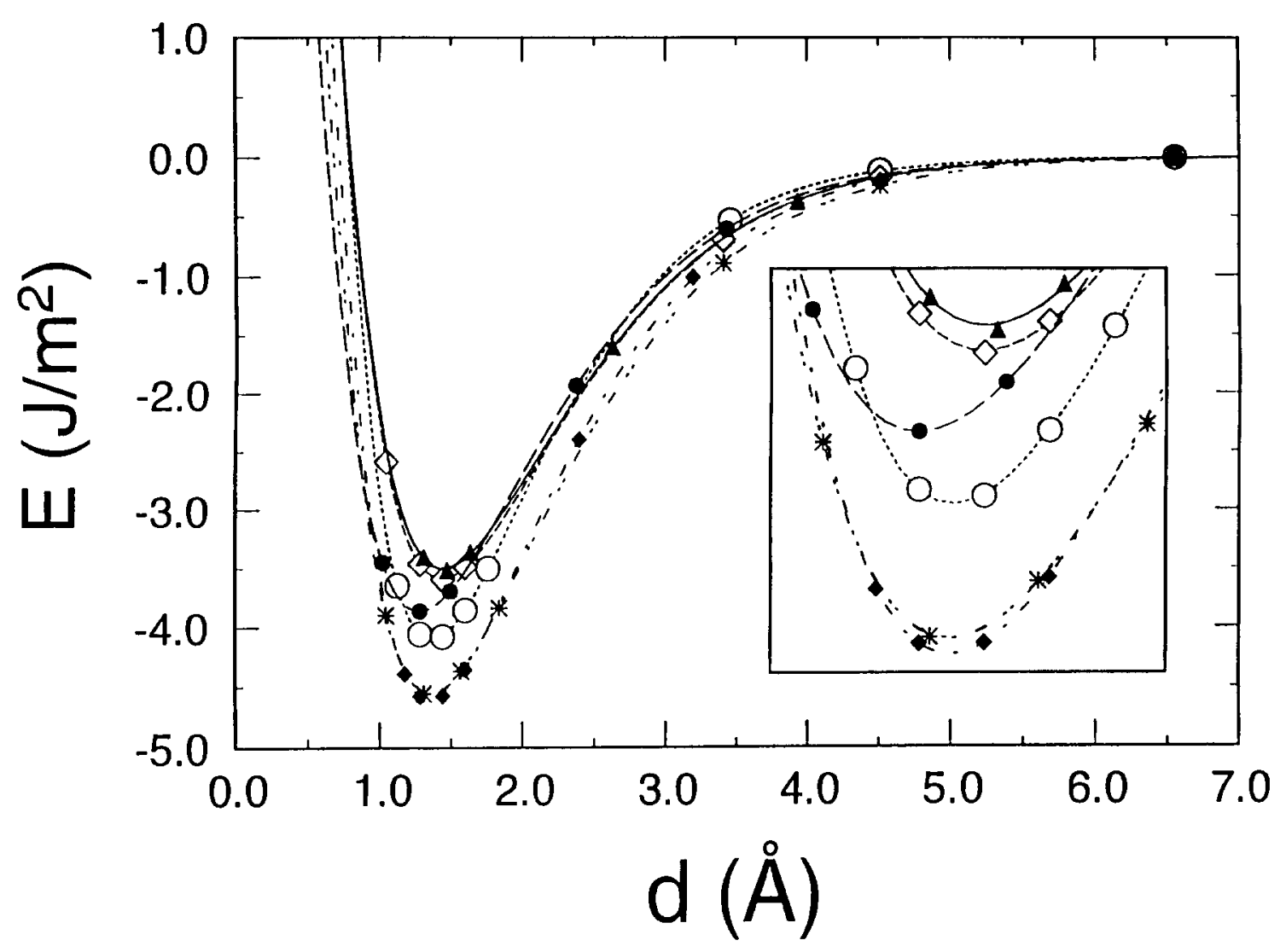

Figure 2. The calculated total energy $E$ (per unit surface area) at different interfacial separations and the corresponding universalbinding-energy relation (UBER) versus interfacial separation $d$ for all the cases. The inset shows the details in the vicinity of the minima. The o's correspond to MoMo//SiMoSi, $\diamond$ 's to MoMo//SiMoSiSi, 4 's to MoMoMo//SiMoSiSi, $\$$ 's to MoMo//SiSiMoSi, - to MoSi//SiMoSi, and "'s to MoSiSi//MoSiSi. Stacking configurations all start at the mirror plane. Actual slabs contain twice the number of layers listed.

ble 1 , to unequivocally establish which Mo(001)/ $\mathrm{MoSi}_{2}(001)$ interface (i.e., MoMo//SiMoSiSi or $\mathrm{MoMo} /(\mathrm{SiSiMoSi})$ has the lowest energy and hence is thermodynamically stable. A comparison of results for the $\mathrm{MoSi} / / \mathrm{SiMoSi}$ and $\mathrm{MoSiSi} / \mathrm{MoSiSi}$ interfaces shows that the MoSi//SiMoSi represents the lower energy configuration for the free $\mathrm{MoSi}_{2}$. If we were to bring this equilibrium $\mathrm{MoSi}_{2}$ surface in contact with a Mo surface at sufficiently low temperatures, then we would expect the stacking to remain unchanged, i.e., MoMoMo//SiMoSiSi. If the energy difference between MoMoMo//SiMoSiSi and MoMoMo//SiSiMoSi were sufficiently small, then it would be likely that in any $\mathrm{Mo}(001) / / \mathrm{MoSi}_{2}(001)$ composite both interfaces would be present. This assertion is based upon the nonequilibrium nature of composite processing and the manner in which interfaces migrate. Therefore, when the composite is subjected to an external stress which tends to pull the interface apart, it is the weaker interface which would dominate the adhesion properties of material. Examination of table 1 demonstrates that the MoMoMo//SiMoSiSi interface is substantially weaker than the MoMoMo//SiSiMoSi interface, based on both the adhesive energy $E_{0}$ and the peak interfacial strength $\sigma_{\max }$. For all of these reasons we will focus on the Mo$\mathrm{MoMo} / / \mathrm{SiMoSiSi}$ interface for the remainder of the present study.

In order to examine the validity of the universal binding energy relation (UBER) (equation 1) for adhesion between two dissimilar metallic materials, the UBER form is fitted to the calculated 


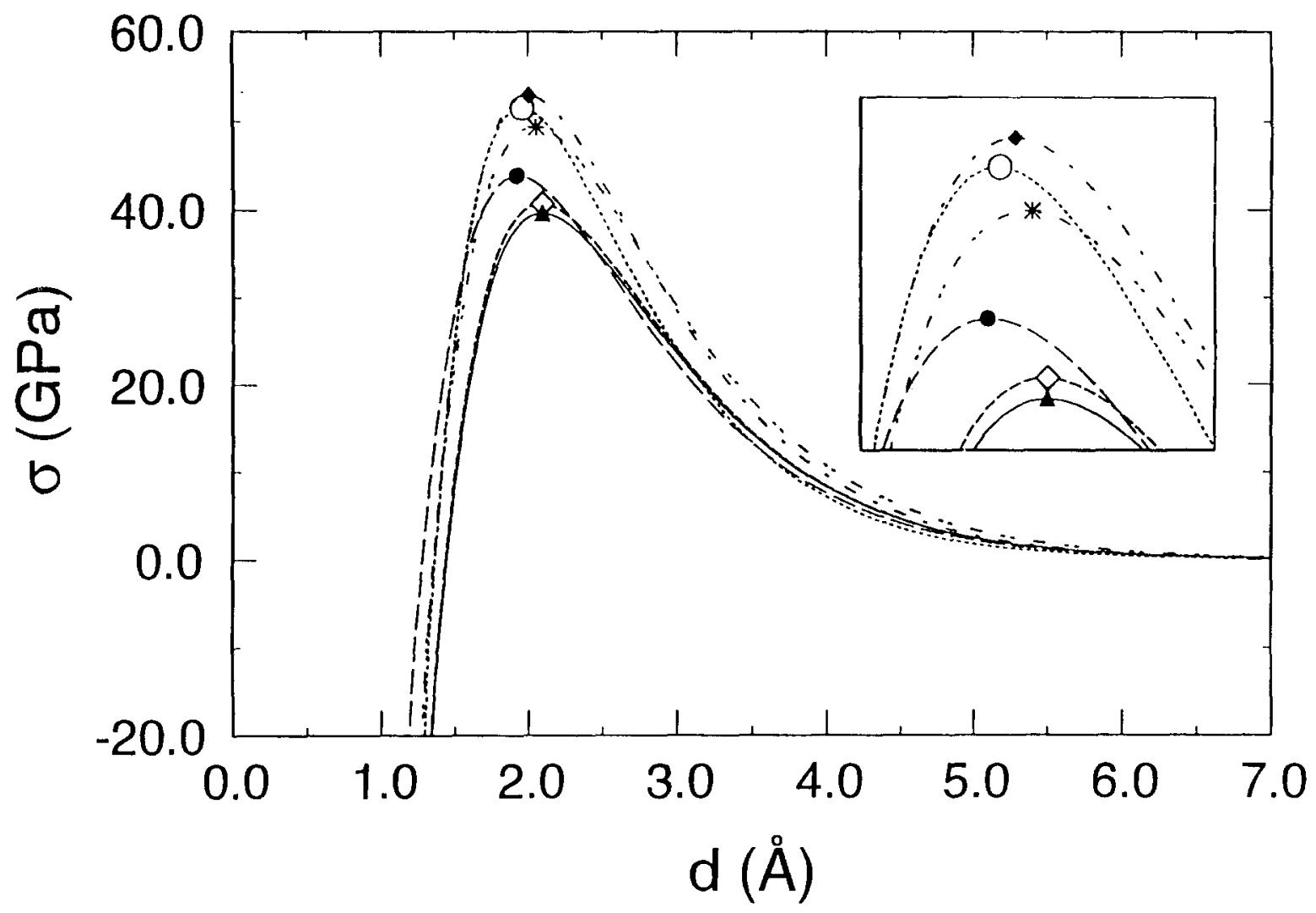

Figure 3. The calculated interfacial stress (per unit cross-sectional area) versus interfacial separation. The marks denote the peak interfacial strength for all of the cases examined. The o corresponds to $\mathrm{MoMo} / \mathrm{SiMoSi}, \diamond$ to $\mathrm{MoMo} / / \mathrm{SiMoSiSi} \Lambda$, to $\mathrm{MoMoMo} / \mathrm{SiMoSiSi}$, to MoMo//SiSiMoSi, $\bullet$ to MoSi//SiMoSi, and * to MoSiSi//MoSiSi.

energy values at different interfacial separations and plotted in figure 2 for the six interfaces analyzed in table 1 . In all cases, the UBER provides an excellent fit to the data. The MoMo//SiMoSiSi and the MoMoMo//SiMoSiSi adhesion curves are very close over the entire range of the interfacial separation, reflecting the short-screening effect in Mo, as discussed earlier. Strikingly, the adhesion curves for the MoMo//SiSiMoSi and MoSiSi//MoSiSi interfaces are nearly the same over a wide range of the interfacial separation. This suggests that the /MoSiSi surface energy is similar to that of the pure Mo (001) regarding adhesion.

For completeness, the calculated interfacial stress is plotted as a function of interfacial separation in figure 3 for all of the interfaces listed in table 1 . While the stress-separation curves for the probable interface configuration $\mathrm{MoMo} / \mathrm{SiMoSiSi}$ and MoMoMo//SiMoSiSi are nearly identical, the MoMo//SiMoSiSi and MoMo//SiSiMoSi stress-separation curves are quite different. The probable interfacial arrangement $\mathrm{MoMo} / / \mathrm{SiMoSiSi}$ has both a smaller adhesive energy and interfacial strength than the $\mathrm{MoMo} / \mathrm{SiSiMoSi}$ interface or the (001) planes of single-crystal $\mathrm{MoSi}_{2}$ and Mo [4].

\subsection{Interfacial electron redistribution}

When two materials are brought into adhesive contact, electron rearrangement occurs at the interface. The electron rearrangement is normally associated with the formation of adhesive bonds. In order to compare the role of electron rearrangement in different interfacial geometries, we plot the changes in the electron density profiles for the MoMo//SiMoSiSi interface, the MoMo//SiSiMoSi interface, and perfect 


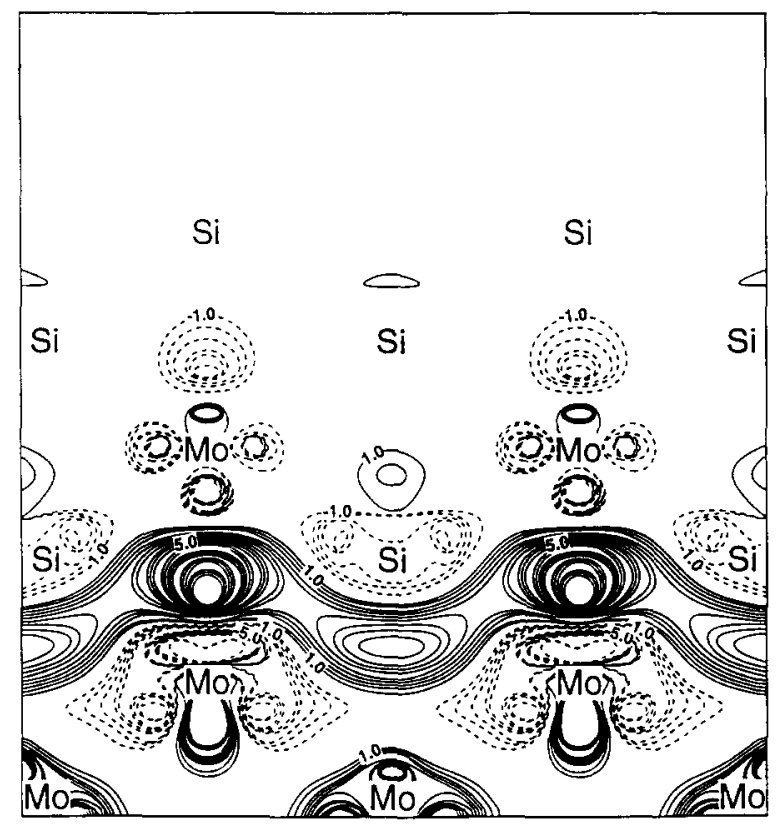

(a)

$\mathrm{MoSi}_{2}$ (MoSi//SiMoSi) when they are formed by bringing the two slabs together from large separations (figures $4(\mathrm{a}-\mathrm{c})$, respectively). These plots are generated by subtracting the electron charge density distributions at the equilibrium interfacial separation by those at large separation. Examination of the electron rearrangement shown in figures $4(a-c)$ demonstrates that charge rearrangement is effectively limited to one or two atomic layers in all three cases. This provides further direct evidence of the short screening lengths associated with these metallic systems.

The magnitude of the electron rearrangement associated with the formation of the adhesive bonds is quite strong at the interface. The characteristics of the electron rearrangement are distinct in each of the three cases. In the perfect $\mathrm{MoSi}_{2}$ crystal (MoSi//SiMoSi) (figure 4(c)), electrons accumulate along the interface midway between the Si and Mo atoms. These electrons are mainly drawn from the regions near the $\mathrm{Si}$ and Mo atoms, which were on the surfaces prior to the formation of the adhesive bond. This is very similar to the formation of covalent bonds in diatomic molecules [5] in which the atoms

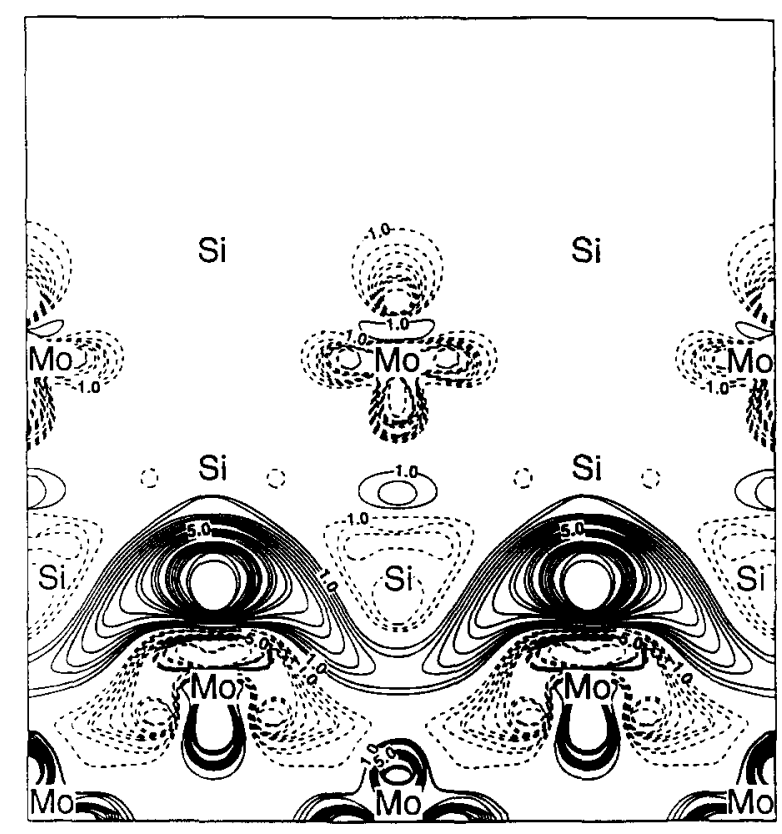

(b)

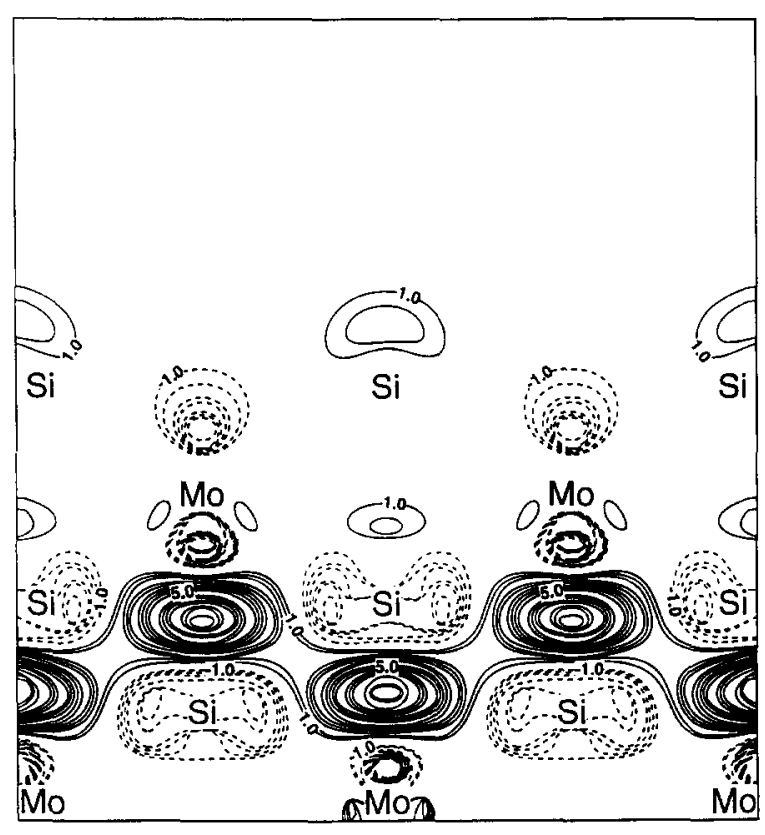

(c)

Figure 4. Electron rearrangement associated with creating an interface projected onto the (110) plane caused by ideal adhesion in (a) MoMo//SiMoSiSi; MoMo//SiSiMoSi; and $\mathrm{MoSi} / / \mathrm{SiMoSi}$. The numbers in all the figures are in units of $10^{-3}$ electron/(a.u.) ${ }^{3}$. Positive contours (solid lines) denote electron accumulation due to ideal adhesion and negative contours (dashed lines) indicate electron depletion. 
involved in bonding contribute electrons to the interatomic regions where bonds are formed. It is interesting to note that no substantial electron accumulation occurs between the $\mathrm{Si}$ atoms across the interface, even though they are closer than the Mo-Si pairs. This indicates that the Mo-Si bonding dominates in crystalline $\mathrm{MoSi}_{2}$.

In addition to the electron rearrangement characterized by the atoms at the interface contributing electrons to the interfacial region in the $\mathrm{MoSi} / / \mathrm{SiMoSi}$ case (figure 4(c)), same-site electron rearrangement between states of different symmetry also takes place at the MoMo//SiMoSiSi (figure 4(a)) and MoMo//SiSiMoSi (figure 4(b)) interfaces. It is clear that the Mo atoms at the interface lose some electrons in the $d_{x z}$ and $d_{y z}$ states while gaining electrons in the $d_{z^{2}-r^{2}}$ state. This obviously tends to strengthen the adhesive bonds. In the MoMo//SiMoSiSi case, the electron accumulation between the Mo and $\mathrm{Si}$ atoms at the interface is not as strong as in the pure $\mathrm{MoSi}_{2}$ (MoSi//SiMoSi) case. On the other hand, the electron rearrangement between the Mo atoms on the Mo side of the interface and the Mo atoms two layers away across the interface are greatly enhanced. In addition to the strong MoMo bonds formed at the interface, there is also substantial electron accumulation between the $\mathrm{Mo}$ and $\mathrm{Si}$ atoms at the interface. This helps to form a substantial, continuous band of electron accumulation along the interface. Thus, the adhesive bonds at the MoMo/SiMoSiSi interface are best described as the combination of a uniform band of electron accumulation along the interface and directional electron accumulations between the atoms across the interface along the direction perpendicular to the interface.

The MoMo//SiSiMoSi interface exhibits yet another type of adhesive bond. In this case (figure 4(b)), there are strong electron accumulations between the Mo atoms at the interface and the two Si layers across the interface. The electron accumulations in the interatomic regions between the subsurface Mo and the interfacial Si atoms is small. Note that the electron rearrangement around the Mo atoms in $\mathrm{MoSi}_{2}$ and the second layer Mo atoms in the Mo slab is larger than the comparable sites in the MoMo//SiMoSiSi case (figure 4(a)). While the above discussion focusing on electron rearrangement due to ideal adhesion can be useful in elucidating the characteristics of adhesive bonds, quantitative connections between the adhesive energetics and the electron rearrangement require total energy computations, as provided in Section 3.1.

\subsection{Interfacial density of states}

As illustrated above, electron rearrangement caused by ideal adhesion is essentially limited to within one or two atomic layers of the interface. It is therefore natural to examine the changes in electronic structure caused by the interfacial contact by focusing on the atomic layers at the interface. Figures $5(\mathrm{a}$ and $\mathrm{b})$ show the local densities of states (LDOS) for atoms at the MoMo//SiMoSiSi and MoMo//SiSiMoSi interfaces, respectively, where the zero of the energy scale was chosen as the Fermi energy. The plots are for the equilibrium interfacial separation. The upper panels in figures $5(\mathrm{a}$ and $\mathrm{b})$ show the total LDOS on the atomic sites within one layer on either side of the interface. The lower panel shows the LDOS at the same sites, where the bulk-like states have been subtracted out. These are referred to as interface states, which are defined as those states with more than $55 \%$ of their weight concentrated on the two layers at the interface. For comparison, the LDOS for the center layer of a five-layer Mo slab and the three center layers of the MoSi//SiMoSi system which contain one formula unit of $\mathrm{MoSi}_{2}$ are also shown in figures 5(c and d), respectively. These layers are supposedly bulk-like layers in the slabs and, indeed, figures $5(\mathrm{c}$ and $\mathrm{d}$ ) are in very good agreement with those for bulk Mo and $\mathrm{MoSi}_{2}$ from earlier first-principles studies [11-14]. This demonstrates, from an electronic structure point of view, that relatively thin slabs can be used to represent bulk metallic materials. The LDOS for atoms at the $\mathrm{MoSiSi} / / \mathrm{MoSiSi}$ interface are not shown as they are essentially what are expected for bulk-like $\mathrm{MoSi}_{2}$ atoms, as shown in figure 5(d) and previously published studies [13, 14]. Although there is no unique way of projecting the LDOS onto atomic orbitals located on individual planes, the widely used Mulliken [15] 


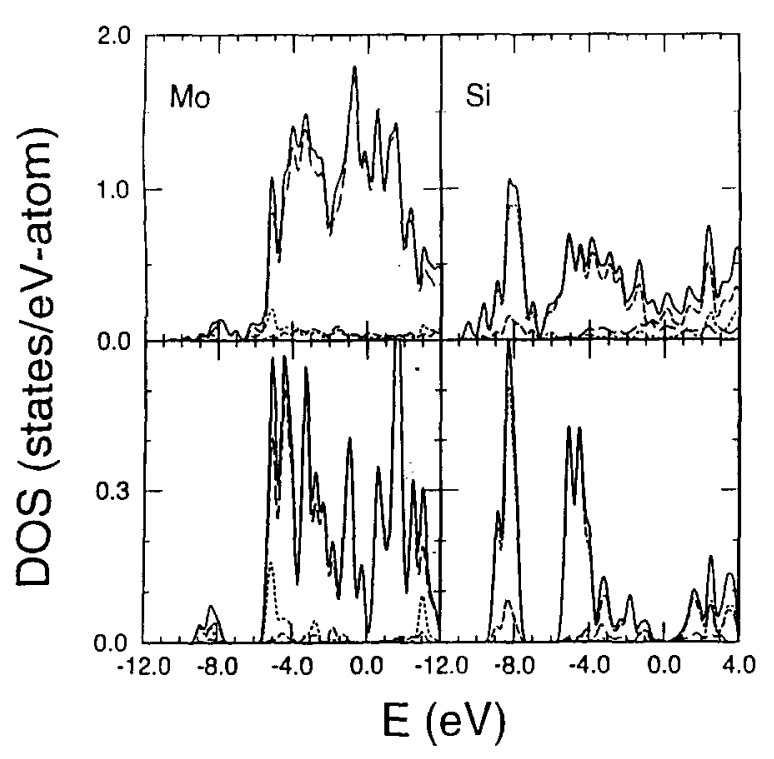

(a)

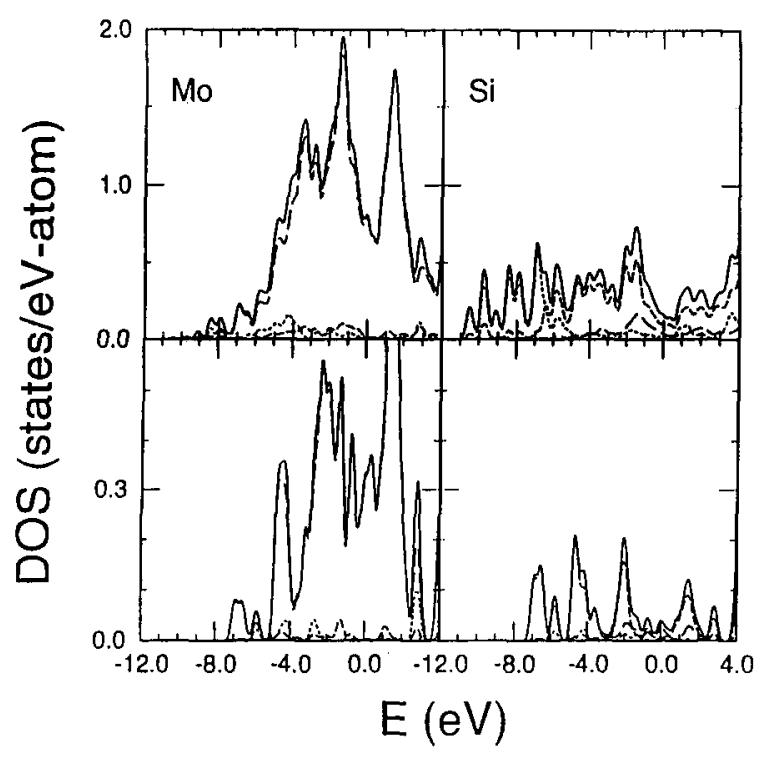

(b)

projection technique is employed to obtain the projected LDOS in figures 5(a-d). All the LDOS are broadened by Gaussians with a full width at half maximum of $0.4 \mathrm{eV}$.

In the MoMo//SiMoSiSi case (figure 5(a)), the LDOS at the Mo site at the interface are intermediate between those in crystalline Mo (fig-

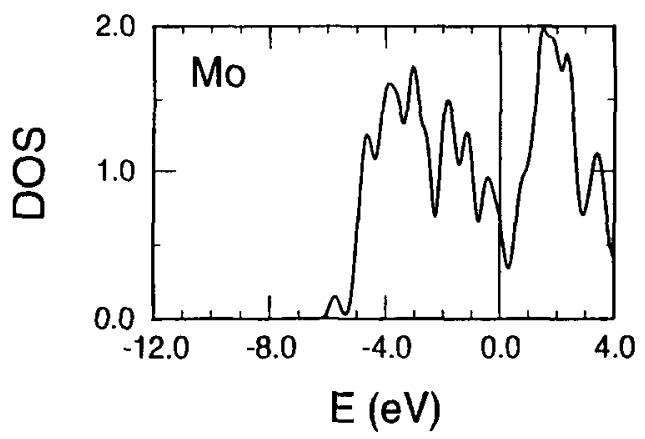

(c)

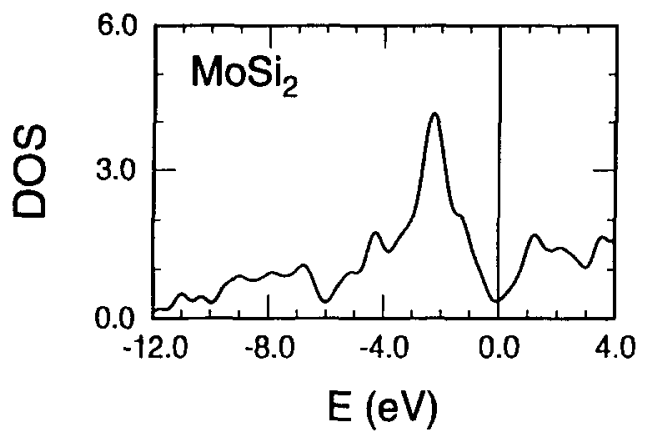

(d)

Figure 5. The calculated local densities of states (LDOS) on Mo atoms on the Mo side of the interface and the $\mathrm{Si}$ atoms adjacent to the interface on the $\mathrm{MoSi}_{2}$ side of (a) MoMo//SiMoSiSi and (b) MoMo//SiSiMoSi are displayed. The plots are for the equilibrium interfacial separation. The layers are labeled by element. The upper panel of each figure shows the total LDOS, while the LDOS due to interface states are plotted in the lower panel. Note the change of scale between the upper and lower panels. Solid lines give the LDOS from orbitals with all symmetry, and dotted, dashed, and long-dashed lines represent the LDOS from $s-, p$ - , and $d$-orbitals, respectively. The LDOS for (c) the center Mo layer of a five-layer Mo slab and the (d) three center layers of the MoSi//SiMoSi system are also shown.

ure $5(\mathrm{c})$ or $[11,12])$ and those for $\mathrm{Mo}$ in $\mathrm{MoSi}_{2}$ (the profile of which is primarily as that is shown in figure 5(d), see $[13,14])$ However, the deep valley (low LDOS region) near the Fermi energy for the Mo sites in both crystalline Mo and $\mathrm{MoSi}_{2}$, which is generally associated with the stable phase, is not present in the LDOS. Consequently, the $4 d$ LDOS at the Fermi energy is high. The $3 s$ orbital at the interfacial $\mathrm{Si}$ sites is localized within a narrow energy range centered at $-8 \mathrm{eV}$, in contrast to the situation in 
crystalline $\mathrm{MoSi}_{2}$, where the $3 s$ orbital spreads over a wide energy range approximately between -13 and $-6 \mathrm{eV}$. This suggests that the interfacial $\mathrm{Si} 3 s$ orbital is not actively involved in the hybridization of orbitals in the MoMo//SiMoSiSi case. There are groups of peaks dominated by the Mo- $4 d$ and the $\mathrm{Si}-3 p$ orbitals at the $\mathrm{Mo}$ and $\mathrm{Si}$ sites at the interface, respectively, between about -6 and $-2 \mathrm{eV}$. These peaks are well separated from the states at higher energies and presumably constitute a large fraction of the bonding states. The Mo-4d peaks between -2 and $2 \mathrm{eV}$ do not have prominent counterparts from the $\mathrm{Si}$ $3 p$ orbitals in the same energy range. To some extent, the LDOS due to interface states in the lower panel merely echo the corresponding total LDOS. The peaks that occur between -6 and $-2 \mathrm{eV}$ are separated by a deep valley at about $-3.7 \mathrm{eV}$ at the interfacial Mo site, while only the lower part remains significant at the interfacial Si site. The LDOS values at the Fermi energy at both the interfacial Mo and Si sites are very small.

The LDOS at the interfacial Mo and Si sites in the MoMo//SiSiMoSi case are not much different from those in crystalline $\mathrm{MoSi}_{2}$ (figure 5(d)) and $[13,14])$. Both the peak between -2 and $-1 \mathrm{eV}$ and the peak at about $2 \mathrm{eV}$ at the interfacial Mo have counterparts in $\mathrm{MoSi}_{2}$. A deep valley at the Fermi energy is also reminiscent of the situation in $\mathrm{MoSi}_{2}$. The most significant difference between the MoMo//SiSiMoSi and crystalline $\mathrm{MoSi}_{2}$ cases is a peak centered at about $-3.5 \mathrm{eV}$ attributable to the Mod-Mod hybridization which is missing in $\mathrm{MoSi}_{2}$. The Si $3 s$ orbital, in the MoMo//SiSiMoSi interfacial case, occupies a wide energy range comparable to that in $\mathrm{MoSi}_{2}$ owing to the Sis-Sis hybridization, in contrast with MoMo//SiMoSiSi case (figure 5(a)). The overall resemblance of the LDOS between the $\mathrm{MoMo} / / \mathrm{SiSiMoSi}$ interface and crystalline $\mathrm{MoSi}_{2}$ is consistent with the results of adhesive energetics in these two cases, as discussed earlier. The strong binding (larger ideal adhesive energy) in the MoMo//SiSiMoSi case is presumably due to MoSiSi//MoSiSi having an $E_{0}$ which is larger than that of $\mathrm{MoSi} / \mathrm{SiMoSi}$.

It is informative to look at the band structure of interface states as well as their LDOS shown above. Figures $6(a$ and $b)$ exhibit the energy bands attributable to interface states (defined earlier) for the MoMo//SiMoSiSi and MoMo//SiSiMoSi cases, respectively. The distributions of interface states in the two cases are quite different. In the MoMo//SiMoSiSi case, widely distributed interface states exist with modest weight at the interface between -9 and $-8 \mathrm{eV}$ which are primarily attributable to the Si $3 s$ orbital. These interface states are separated from others at higher energies by more than $2 \mathrm{eV}$, which again indicate weak hybridization between the Si $3 s$ and other orbitals. On the other hand, the interface states dominated by the $\mathrm{Si} 3 s$ are essentially limited to the $Y$ direction in the MoMo//SiSiMoSi case. These interface states are highly concentrated (indicated by the large circle sizes) on the two interface layers and are distributed around $-7 \mathrm{eV}$. In addition, they are not as widely separated from the other interface states as in the MoMo//SiMoSiSi case.

At higher energies (above $-5 \mathrm{eV}$ ), both the MoMo//SiMoSiSi and MoMo//SiSiMoSi cases show a large number of interface states dominated by the Mo $4 d$ and the Si $3 p$. It is intriguing to note that the interface states in the MoMo//SiSiMoSi case tend to have higher concentration at the interface (as shown by larger circle sizes) than those in the MoMo//SiMoSiSi case. While there are highly localized interface states near the $\Gamma$ point at about $-3 \mathrm{eV}$ and the $\mathrm{X}$ point between $-2 \mathrm{eV}$ and the Fermi energy in the MoMo//SiSiMoSi case, few of these interface states appear in the MoMo//SiMoSiSi case. Just below $2 \mathrm{eV}$, highly localized interface states are shown in both cases. These are centered at the $\mathrm{X}$ and $\mathrm{M}$ points in the MoMo//SiMoSiSi case and are primarily distributed along the $Y$ direction in the MoMo//SiSiMoSi case.

\section{Summary}

A first-principles study of interfacial energetics, bonding characteristics, and electronic structure associated with ideal adhesion at the $\mathbf{M o}-\mathbf{M o S i}_{2}$ (001) heterophase interface has been presented. Results from this study suggested that the interfacial bonding mechanisms are strongly dependent upon the interfacial arrangements (i.e., the stacking sequence). The adhesion between the Mo 


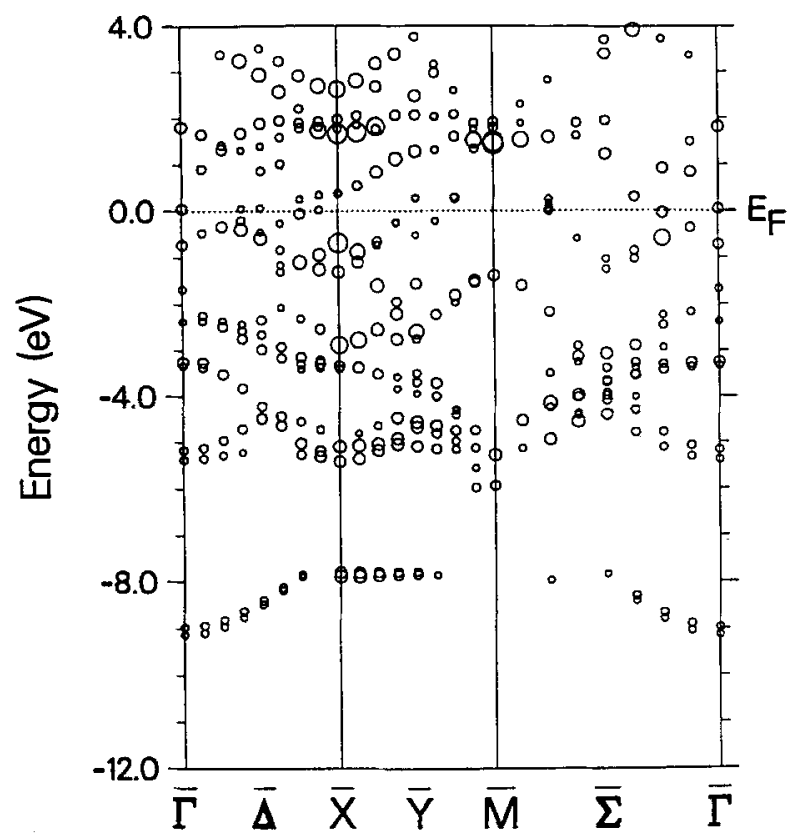

(a)

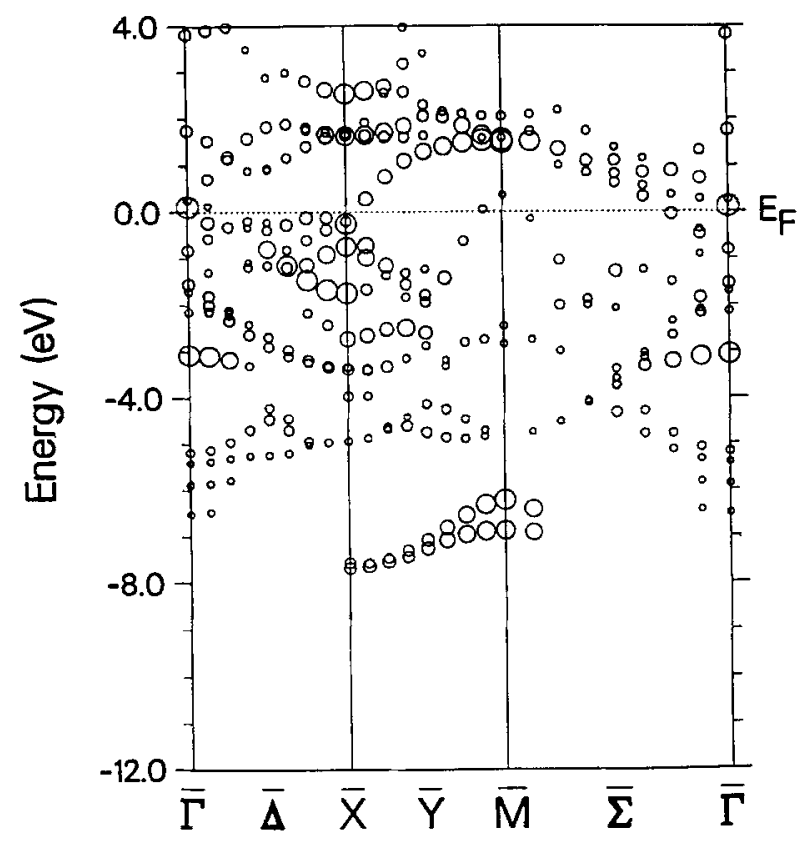

(b)

Figure 6. The calculated energy bands due to interface states, as defined in the text, for (a) MoMo//SiMoSiSi; and (b) $\mathrm{MoMo} / / \mathrm{SiSiMoSi}$. The sizes of the circles are directly proportional to the interface state electron densities on atoms at the interface.

and $\mathrm{Si}$ layers was shown to be stronger than the adhesion between two $\mathrm{Si}$ layers in crystalline $\mathrm{MoSi}_{2}$. Thus the lower surface energy stacking for the $\mathrm{MoSi}_{2}$ free surface is a single $\mathrm{Si}$ at the surface with a Mo layer beneath it. Bringing this surface stacking in contact with the Mo yields a MoMo//SiMoSiSi stacking, which we consider to be the likely equilibrium stacking. The $\mathrm{MoMo} / / \mathrm{SiMoSiSi}$ was shown to have weaker adhesion than the MoMo//SiSiMoSi interface and, thus, is likely to dominate the adhesion properties of the material consisting of both Mo and $\mathrm{MoSi}_{2}$. The adhesion in the interfacial configuration in which $\mathrm{MoSi}_{2}$ has two Si layers at the interface (MoMo//SiSiMoSi) was shown to resemble the adhesion between Mo and Si layers in crystalline $\mathrm{MoSi}_{2}$. Stronger adhesion found at the MoMo//SiSiMoSi configuration is therefore attributable to the higher energy needed to create the $\mathrm{MoSi}_{2}(001)$ free surface with two $\mathrm{Si}$ layers at the surface. Both the ideal adhesive energy and peak interfacial strength for the
MoMo//SiMoSiSi interface are between $10 \%$ and $15 \%$ smaller than those for crystalline $\mathrm{Mo}(001)$ and $\mathrm{MoSi}_{2}(001)$. The equilibrium interlayer separation between $\mathrm{Mo}$ and $\mathrm{MoSi}_{2}$ in the interfacial arrangement was found to be intermediate between those in crystalline $\mathrm{Mo}$ and $\mathrm{MoSi}_{2}$. The adhesive bonding at the stable interface was identified to be a combination of a uniform band of charge accumulation at the interface and directional charge accumulation between atoms across the interface.

\section{Acknowledgments}

The authors are indebted to Drs. J.G. Gay and R. Richter for many fruitful conversations. They would also like to thank Drs. R. Najafabadi and J.M. Rickman for enlightening discussions regarding interfacial configurations that helped us to interpret our results. T.H. and D.J.S. grate- 
fully acknowledge the support of the Air Force Office of Scientific Research, grant AFOSR-900112, under whose auspices this work was performed. All of the authors wish to thank the excellent support and service of the San Diego Supercomputer Center and the Wright-Patterson Air Force Base Supercomputer Center.

\section{References}

1. A.A. Griffith, Phil. Trans. R. Soc. A221, 163 (1920).

2. P. Hohenberg and W. Kohn, Phys. Rev. 136, 864 (1964); W. Kohn and L.J. Sham, Phys. Rev. 140, A1133 (1965).

3. J.R. Smith, J.G. Gay, and F.J. Arlinghaus, Phys. Rev. B 21, 2201 (1980).

4. T. Hong, J.R. Smith, D.J. Srolovitz, J.G. Gay, and R. Richter, Phys. Rev. B 45, 8775 (1992).

5. A. Banerjea and J. R. Smith, Phys. Rev. B 37, 6632 (1988); see also P. Vinet, J.H. Rose, J. Ferrante, and J.R. Smith, J. Phys. 1, 1941 (1989).
6. D. Miracle, J. Graves, and D. Anton, eds. Intermetallic Matrix Composites II (Materials Research Society, Pittsburgh, PA 1992).

7. P. Villars and L.D. Calvert, Pearson's Handbook of Crystallographic Data for Intermetallic Phases (American Society for Metals, Metals Park, OH, 1985).

8. C. Kittel, Introduction to Solid State Physics, 6th Ed. (Wiley, New York, 1986), p. 57.

9. S.H. Vosko, L. Wilk M. Nusair, Can. J. Phys. 58, 1700 (1980); D.M. Ceperley, B.J. Alder, Phys. Rev. Lett. 45, 566 (1980).

10. J.R. Smith, J. Ferrante, P. Vinet, J.G. Gay, R. Richter, and J.H. Rose, in Chemistry and Physics of Fracture, edited by R.M. Latanision and R.H. Jones, NATO ASI Series, Series F: Applied Sciences, No. 130 (Nijhoff, Boston, 1987).

11. V.L. Moruzzi, J.F. Janak, and A.R. Williams, Calculated Electronic Properties of Metals (Pergamon, New York, 1978).

12. S.R. Chubb, E. Wimmer, A.J. Freeman, J.R. Hiskes, and A.M. Karo, Phys. Rev. B 36, 4112 (1987).

13. B.K. Bhattacharya, D.M. Bylander, and L. Kleinman, Phys. Rev. B 32, 7973 (1985).

14. Shaoping Tang and Kaiming Zhang, J. Phys. C: Solid State Phys. 21, 1469 (1988).

15. R.S. Mulliken, J. Chem. Phys. 23, 1833 (1955) 